\title{
Clustered natural frequencies in multi-span beams with constrained characteristic functions
}

\author{
Khodabakhsh Saeedi* and Rama B. Bhat \\ Mechanical and Industrial Engineering Department, Concordia University, Montreal, QC, H3G1M8, Canada
}

Received 24 December 2008

Revised 13 October 2009

\begin{abstract}
A study of the natural frequencies and mode shapes of a multi-span beam is carried out by introducing constrained beam characteristic functions. The conventional method used for the dynamic analysis of such a beam is to consider span-wise characteristic function solution and then to solve it by using compatibility conditions such as the continuity in the a slope and bending moment at the intermediate supports and boundary conditions at the ends. In the method proposed here, the matrix size is reduced and, if the support conditions are symmetric about the midpoint, the symmetry and anti-symmetry conditions at the midpoint can be conveniently exploited for computational economy. The natural frequencies occur in clusters, each one containing the number of natural frequencies equal to the number of spans. The results are presented and discussed.
\end{abstract}

Keywords: Multi-span beam, vibration, intermediate support, reaction force

\section{Introduction}

Vehicle loading on multi-span bridges is more and more assuming an enormous interest in view of the need for infrastructure renewal. Bridges may be modeled as multi-span beams in a preliminary analysis. Although extensive research has been carried out on the dynamic analysis of beams, such as important vibration aspects, vibration analysis of multi-span beams has not received enough attention.

Lin [1] has studied the natural frequencies and mode shapes of a beam with uniformly spaced supports. In this study supports are modeled as either elastic with finite stiffness springs or rigid with the spring stiffness tending to infinity. The effects of the random deviation of the span lengths from the ideal design have been studied by Yang and Lin [2] using the perturbation method. Also the vibration analysis of a plate over intermediate stringers has been studied by Lin et al. [3]. Mead and Yaman [4] have investigated the harmonic response of a multi-span beam excited by single or multi-point harmonic forces by using the wave propagation approach. The results of these studies have been widely used by Memory et al. [5] in practical structures such as bridges. How a multi-support beam as a bridge responds structurally to seismic excitation has been investigated by Loh and $\mathrm{Ku}$ [6]. The reduction of transmitted power in a finite periodic beam has been studied by $\mathrm{Wu}$ and White [7].

The influence of moving loads on bridges or railways is modeled using multi-span beams. The vibration analysis of a beam subjected to a moving mass has been investigated by Ichikawa et al. [8]. They modeled a bridge as a multi-span Euler-Bernoulli beam and obtained analytical expressions for the mode shapes by dividing it into some single-span beams that satisfy compatibility conditions in constraints. These orthogonal eigen-functions were used to represent the inertia terms caused by the motion of the moving mass. A similar approach was followed

*Corresponding author. Tel.: +1 5148482424 Ext. 7268; E-mail: k_saeedi@encs.concordia.ca. 
$L$

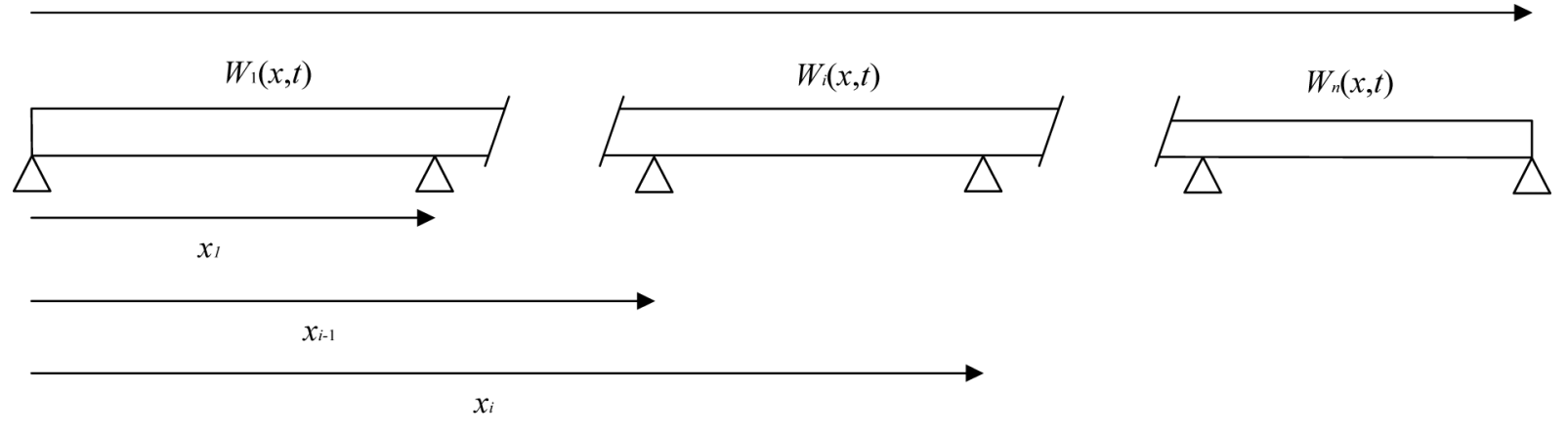

Fig. 1. Multi-span beam with simple intermediate supports.

to investigate the effects of a moving force on such beams by Zhu and Law [9]. In addition, Lin and Chang [10] have investigated the free vibration analysis of a multi-span Timoshenko beam supported by an arbitrary number of flexible constraints by separating it into different parts and by using a transfer matrix method to connect the boundary conditions and mode shapes of each individual part to the whole system. Zhu and Law [11] and Naguleswaran [12], using a procedure similar to that mentioned before, have investigated the dynamic behaviour of an Euler-Bernoulli beam with elastic bearings.

Recently, Lin and Tsai [13] studied the vibration of the uniform multi-span Euler-Bernoulli beam. Yesilce and Demirdag [14] studied a Timoshenko beam carrying multiple spring mass systems. In these studies the beam was divided into parts. The compatibility conditions as well as the dynamic equilibrium at the support constraints and spring-mass attachments were taken into account. It has been shown that these procedures generally lead to orthogonal mode shapes [15].

Although splitting a multi-span beam into single-span beams is an accurate method leading to the exact solution of the eigen-problem, such a procedure usually generates a large eigen-problem especially when dealing with a big number of spans, because each compatibility condition such as the continuity in the slope and bending moment, should be satisfied at each support. The method, proposed in this study, treats the multi-span beam as a single beam with forces acting on the supports. In this method, the deflection of the beam has been defined by one function and the constraint reactions at the supports are included in the solution of the eigen-value problem. It is shown that fewer mathematical operations are needed in order to obtain the same results as those obtained by the conventional span-wise characteristic function method. Moreover, there is no need to satisfy conditions such as the continuity in the bending moment and slope because the deflection is defined by one function.

\section{Span-wise characteristic function method}

A brief review of the conventional span-wise characteristic function method is given below. For the multi-span beam shown in Fig. 1, the equations of motion can be written for each span with appropriate end boundary conditions and compatibility conditions at the intermediate supports. The equations of motion of the beam can be written as [8]:

$$
E I W_{i}^{I V}(x, t)+\rho A \ddot{W}_{i}(x, t)=0 \quad x_{i-1} \leqslant x \leqslant x_{i} \quad \forall i=1,2, \ldots, n
$$

where $W_{i}(x, t)$ represents the beam deflection in the $i^{t h}$ span.

Assuming harmonic motion under free vibration conditions, the solution may be written as $W_{i}(x, t)=\psi_{i}(x) e^{i \omega t}$ and the deflection can be expressed in the following form $[8,13]$ :

$$
\psi_{i}(x)=B_{1 i} \sin \beta x+B_{2 i} \cos \beta x+B_{3 i} \sinh \beta x+B_{4 i} \cosh \beta x \quad x_{i-1} \leqslant x \leqslant x_{i} \quad \forall i=1,2, \ldots, n
$$

where

$$
\beta^{4}=\frac{\rho A \omega^{2}}{E I}
$$


$L$

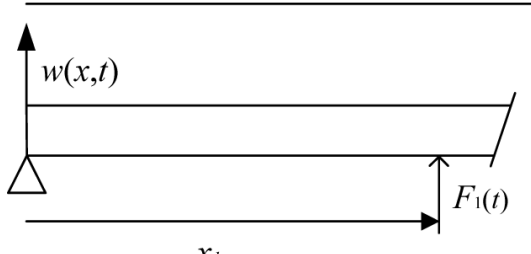

$x_{1}$
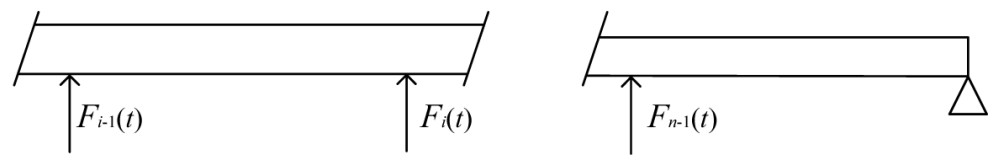

$x_{i-1}$

$x_{i}$

Fig. 2. Multi-span beam with intermediate supports, replaced by time-dependent forces.

which should satisfy the following boundary conditions:

$$
\begin{array}{lcr}
\psi_{i}\left(x_{i-1}\right)=0 & \psi_{i}\left(x_{i}\right)=0 & \forall i=1,2, \ldots, n \\
\psi_{1}^{I I}\left(x_{0}\right)=0 & \psi_{n}^{I I}\left(x_{n}\right)=0 & \\
\psi_{i}^{I}\left(x_{i}\right)=\psi_{i+1}^{I}\left(x_{i}\right) & \forall i=1,2, \ldots, n-1 \\
\psi_{i}^{I I}\left(x_{i}\right)=\psi_{i+1}^{I I}\left(x_{i}\right) & \forall i=1,2, \ldots, n-1
\end{array}
$$

It should be noted that in the equations given above, the values that apply are as: $x_{0}=0$ and $x_{n}=L$. These boundary conditions correspond to vanishing the deflection at the supports, vanishing the bending moment at the both ends of the beam, and two sets of continuity conditions at each intermediate support. A set of algebraic equations with a $(4 \times n)$ unknowns can be built on the base of the boundary conditions, where $B_{i j}(i=1,2,3,4$ and $j=1, \ldots$, $n$ ) form the eigen-vector of the span-wise characteristic function method. The natural frequency, $\omega$, can be obtained by finding the roots of the determinant of the coefficient matrix and then, the ratio of the $B_{i j} / B_{11}$ can be calculated. The mode shapes obtained are also orthogonal functions [15]. The remaining constant is an arbitrary constant which can be defined by normalizing the mode shapes as follows:

$$
\rho A \sum_{i=1}^{n} \int_{x_{i-1}}^{x_{i}} \psi_{i j}(x) \psi_{i k}(x) d x=\delta_{j k}
$$

Finally, the constraint forces at the intermediate supports are obtained by using the following force equilibrium conditions:

$$
f_{i}=E I \psi_{i}^{I I I}\left(x_{i}\right)-E I \psi_{i+1}^{I I I}\left(x_{i}\right) \quad \forall i=1,2, \ldots, n-1
$$

\section{Constrained characteristic function method}

In Fig. 2, all the constraints except the first and the last are replaced by time-dependent forces, which are reactions at the intermediate supports. The equation of motion can be written as follows:

$$
E I w^{I V}(x, t)+\rho A \ddot{w}(x, t)=\sum_{i=1}^{n-1} F_{i}(t) \delta\left(x-x_{i}\right)
$$

The boundary conditions as well as the intermediate support constraints are defined as follows:

$$
\begin{array}{lc}
w(0, t)=0 & w(L, t)=0 \\
w^{I I}(0, t)=0 & w^{I I}(L, t)=0 \\
w\left(x_{i}, t\right)=0 & \forall i=1,2, \ldots, n-1
\end{array}
$$

To solve Eq. (7), the deflection and the indeterminate forces can be replaced by: 


$$
w(x, t)=\phi(x) e^{i \omega t} \quad F_{i}(t)=f_{i} e^{i \omega t}
$$

The characteristic equation used for solving for the mode shapes, natural frequencies and constraint reactions is the following:

$$
E I \phi^{I V}(x)-\rho A \omega^{2} \phi(x)=\sum_{i=1}^{n-1} f_{i} \delta\left(x-x_{i}\right)
$$

which is subject to the following boundary and constraint conditions:

$$
\begin{array}{lc}
\phi(0)=0 & \phi(L)=0 \\
\phi^{I I}(0)=0 & \phi^{I I}(L)=0 \\
\phi\left(x_{i}\right)=0 & \forall i=1,2, \ldots, n-1
\end{array}
$$

The general solution of Eq. (10) can be expressed as the sum of a homogenous solution and a particular solution as follows:

$$
\phi(x)=\phi_{h}(x)+\phi_{p}(x)
$$

where the homogenous solution is:

$$
\phi_{h}(x)=A_{1} \sin \beta x+A_{2} \cos \beta x+A_{3} \sinh \beta x+A_{4} \cosh \beta x
$$

Since the solution of the left hand side of Eq. (10) to the Dirac delta function input, $\delta(x-\eta)$ is given by:

$$
\phi(x)=\frac{1}{2 E I \beta^{3}}[\sinh \beta(x-\eta)-\sin \beta(x-\eta)] u(x-\eta)
$$

the particular solution can be written by using Eq. (14) as follows:

$$
\phi_{p}(x)=\sum_{i=1}^{n-1} \frac{f_{i}}{2 E I \beta^{3}}\left[\sinh \beta\left(x-x_{i}\right)-\sin \beta\left(x-x_{i}\right)\right] u\left(x-x_{i}\right)
$$

Substituting the boundary conditions, stated in Eq. (11), in the general solution (12) will result in an eigen-value problem in which the unknown coefficient of Eq. (13), $A_{i}$, and the constraint reactions, $f_{i}$, appear in the eigenvectors. Since single function is assumed for the deflection shape, $\phi(x)$, the continuity conditions for the slope and the bending moment at the intermediate supports are not required anymore and the size of the eigen-vector is reduced to $n+3$. It should be noted that the following orthogonality conditions are satisfied by the eigen-functions.

$$
\rho A \int_{0}^{L} \phi_{i}(x) \phi_{j}(x) d x=\delta_{i j}
$$

\section{Orthogonal mode shapes}

According to Eq. (10), the equation of motion of the $j^{t h}$ and $k^{t h}$ modes of the multi-span beam can be written, respectively, as:

$$
E I \phi_{j}^{I V}(x)-\rho A \omega_{j}^{2} \phi_{j}(x)=\sum_{i=1}^{n-1} f_{i j} \delta\left(x-x_{i}\right)
$$

and

$$
E I \phi_{k}^{I V}(x)-\rho A \omega_{k}^{2} \phi_{k}(x)=\sum_{i=1}^{n-1} f_{i k} \delta\left(x-x_{i}\right)
$$

Multiplying Eq. (17) by $\phi_{k}(x)$ and Eq. (18) by $\phi_{j}(x)$, integrating them by parts in the interval $(0, L)$ and subtracting the second result from the first, results in: 
Table 1

Two-span beam with unequal span lengths

\begin{tabular}{|c|c|c|c|c|}
\hline \multirow[t]{2}{*}{ Mode No. } & \multicolumn{2}{|c|}{ Conventional method } & \multicolumn{2}{|c|}{ Present method } \\
\hline & $\begin{array}{l}\text { Natural frequency } \\
(\mathrm{rad} / \mathrm{s})\end{array}$ & $\begin{array}{c}\text { Constraint reaction } \\
(\mathrm{kN})\end{array}$ & $\begin{array}{l}\text { Natural frequency } \\
(\mathrm{rad} / \mathrm{s})\end{array}$ & $\begin{array}{c}\text { Constraint reaction } \\
(\mathrm{kN})\end{array}$ \\
\hline 1 & 49.4562 & -37.946 & 49.4564 & -37.946 \\
\hline 2 & 113.7001 & -167.96 & 113.6999 & -167.96 \\
\hline 3 & 189.2880 & 415.08 & 189.2882 & 415.08 \\
\hline
\end{tabular}

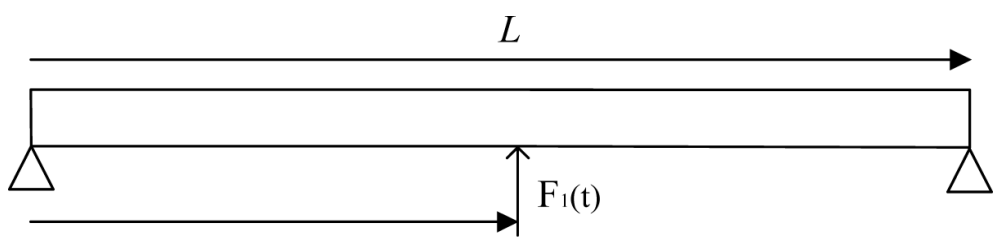

$x_{1}$

Fig. 3. Two-span beam.

$$
\begin{aligned}
& {\left.\left[E I \phi_{j}^{I I I}(x) \phi_{k}(x)-E I \phi_{j}^{I I}(x) \phi_{k}^{I}(x)+E I \phi_{j}^{I}(x) \phi_{k}^{I I}(x)-E I \phi_{j}(x) \phi_{k}^{I I I}(x)\right]\right|_{0} ^{L}} \\
& \quad-\rho A\left(\omega_{j}^{2}-\omega_{k}^{2}\right) \int_{0}^{L} \phi_{j}(x) \phi_{k}(x) d x=\sum_{i=1}^{n-1} f_{i j} \phi_{k}\left(x_{i}\right)-f_{i k} \phi_{j}\left(x_{i}\right)
\end{aligned}
$$

According to the boundary and intermediate support constraint conditions of the beam given in Eq. (11), the right hand side of the equation and the first four terms on the left hand side of the equation are zero. Therefore, the mode shapes of the structure obtained by this method are orthogonal as stated in Eq. (16).

\section{Numerical results}

\subsection{Case study 1}

In this part, the results obtained by the conventional method and the proposed method for the natural frequencies and constraint reaction forces are compared for the case of a two span beam. The beam is shown in Fig. 3. The following values are used:

$$
E I=3.5 \times 10^{6} \mathrm{~N} . \mathrm{m}^{2} \quad \rho A=160 \mathrm{~kg} / \mathrm{m} \quad L=10 \mathrm{~m} \quad x_{1}=6 \mathrm{~m}
$$

The first three natural frequencies of the two-span beam and constraint reactions at the middle support in each mode shape by both methods are presented in Table 1 . As can be seen, all the results obtained by the present method coincide with those arrived at by the conventional method.

\subsection{Case study 2}

In this example, a two-span beam with two equal spans is studied. The eigen-value problem is obtained by substituting $n=2$ and $x_{1}=L / 2$ in Eqs (12), (13) and (15). The constants $A_{2}$ and $A_{4}$ can easily be set to zero by imposing the boundary conditions $\phi(0)=0$ and $\phi^{\prime \prime}(0)=0$. The rest of the unknowns, $f_{1}, A_{1}$ and $A_{3}$ are solved using the conditions $\phi(L)=0, \phi^{\prime \prime}(L)=0$ and $\phi(L / 2)=0$. Finally, the characteristic equation leads to two different equations as follows:

$$
\sin \beta \frac{L}{2}=0 \quad \Rightarrow \quad A_{3}=0 \text { and } f_{1}=0
$$

or

$$
\operatorname{coth} \beta \frac{L}{2}=\cot \beta \frac{L}{2} \Rightarrow f_{1}=4 E I \beta^{3} \cos \beta \frac{L}{2} \mathrm{~A}_{1} \quad \text { and } \quad \mathrm{A}_{3}=-\frac{\sin \beta \frac{L}{2}}{\sinh \beta \frac{L}{2}} \mathrm{~A}_{1}
$$


Table 2

Two-span beam with equal span lengths

\begin{tabular}{ccc}
\hline Mode No. & $\beta \frac{L}{2}$ & $\frac{f_{1}\left(\frac{L}{2}\right)^{3}}{E I A_{1}}$ \\
\hline 1 & $\pi$ & 0 \\
2 & 3.9267 & -171.299 \\
3 & $2 \pi$ & 0 \\
4 & 7.0687 & 998.879 \\
5 & $3 \pi$ & 0 \\
6 & 10.2102 & -3010.491 \\
\hline
\end{tabular}

Table 3

Natural frequencies and support reactions for six-span beam. $\beta^{4}=\frac{\rho A \omega^{2}}{E I}$

\begin{tabular}{|c|c|c|c|c|c|c|c|}
\hline Mode No. & $\beta \frac{L}{6}$ Results of Ref. [1] & $\beta \frac{L}{6}$ & $\frac{f_{1}\left(\frac{L}{6}\right)^{3}}{E I A_{1}}$ & $\frac{f_{2}\left(\frac{L}{6}\right)^{3}}{E I A_{1}}$ & $\frac{f_{3}\left(\frac{L}{6}\right)^{3}}{E I A_{1}}$ & $\frac{f_{4}\left(\frac{L}{6}\right)^{3}}{E I A_{1}}$ & $\frac{f_{5}\left(\frac{L}{6}\right)^{3}}{E I A_{1}}$ \\
\hline 1 & $\pi$ & 3.1416 & 0 & 0 & 0 & 0 & 0 \\
\hline 2 & 3.260 & 3.2605 & -17.633 & 30.494 & -35.190 & 30.468 & -17.591 \\
\hline 3 & 3.557 & 3.5564 & -74.705 & 74.709 & 0 & -74.711 & 74.719 \\
\hline 4 & 3.926 & 3.9266 & -171.305 & 0 & 171.318 & 0 & -171.331 \\
\hline 5 & 4.296 & 4.2975 & -286.736 & -286.804 & 0 & 286.797 & 286.818 \\
\hline 6 & 4.601 & 4.6014 & -380.678 & -659.536 & -761.660 & -659.668 & -380.875 \\
\hline 7 & $2 \pi$ & 6.2832 & 0 & 0 & 0 & 0 & 0 \\
\hline 8 & 6.409 & 6.4098 & 132.72 & 229.82 & 265.352 & 229.82 & 132.72 \\
\hline 9 & 6.708 & 6.7076 & 496.47 & 496.48 & 0 & 496.48 & 496.47 \\
\hline 10 & 7.068 & 7.0685 & 999.08 & 0 & -999.08 & 0 & 999.08 \\
\hline 11 & 7.462 & 7.4295 & 1495.8 & -1495.8 & 0 & -1495.8 & 1495.8 \\
\hline 12 & 7.726 & 7.7269 & 1832.1 & -3174.5 & 3665.9 & -3174.5 & 1832.1 \\
\hline 13 & & 9.4248 & 0 & 0 & 0 & 0 & 0 \\
\hline 14 & & 9.5511 & -439.159 & 760.624 & -878.326 & 760.624 & -439.159 \\
\hline 15 & & 9.8488 & -1572.2 & 1572.2 & 0 & 1572.2 & -1572.2 \\
\hline 16 & & 10.2102 & -3010.4 & 0 & 3010.3 & 0 & -3010.4 \\
\hline 17 & & 10.5716 & -4307 & -4307 & 0 & -4307 & -4307 \\
\hline 18 & & 10.8692 & -5095.8 & -8829.8 & -10321.0 & -8829.8 & -5095.8 \\
\hline
\end{tabular}

A dimensionless parameter, $\beta L / 2$ is introduced as the solution of Eqs (20) and (21). It is shown in Table 2, along with the constraint reaction forces for each mode. As can be seen, the resultant force in the support is zero in the eigen-solutions obtained by Eq. (20), whereas there is a finite reaction force at the support position in the mode-shapes obtained by solving Eq. (21). Results of this straightforward study clearly show that the eigen-vector, obtained by the constrained characteristic function method comprise four unknowns $A_{i}$ as well as the force at the mid-support, $f_{1}$, whereas, the traditional method requires eight unknowns to be solved. Also another advantage is in the constraint force which is expressed analytically and differentiating the mode shapes several times is no longer needed.

\subsection{Case study 3}

Numerical solutions are obtained for a six-span beam. All the constraints are assumed as simply supported, equally dividing the beam. The dimensionless frequency parameters, $\beta L / 6$, are compared with the results presented by Lin [1] in Table 3. Also the support reaction forces at the intermediate constraints are presented. As can be seen, the results coincide with the previous results. Lin [1] reported the existence of clusters of natural frequencies. The number of natural frequencies in each cluster is equal to the number of spans. The occurrence of these clusters of frequencies is also seen in the results obtained by the constrained characteristic function method which is given in Table 3.

The corresponding mode shapes for the first three clusters are shown in Figs 4, 5 and 6 . The mode shapes for Cluster 1 in Fig. 4, contain a single half wave in each span, while the Clusters 2 and 3 in Figs 5 and 6 consist of two and three half waves, respectively, in each span. Also it should be noted that all the odd-numbered mode shapes are anti-symmetric with respect to the mid-span of the beam, while there is symmetry in the even-numbered mode 

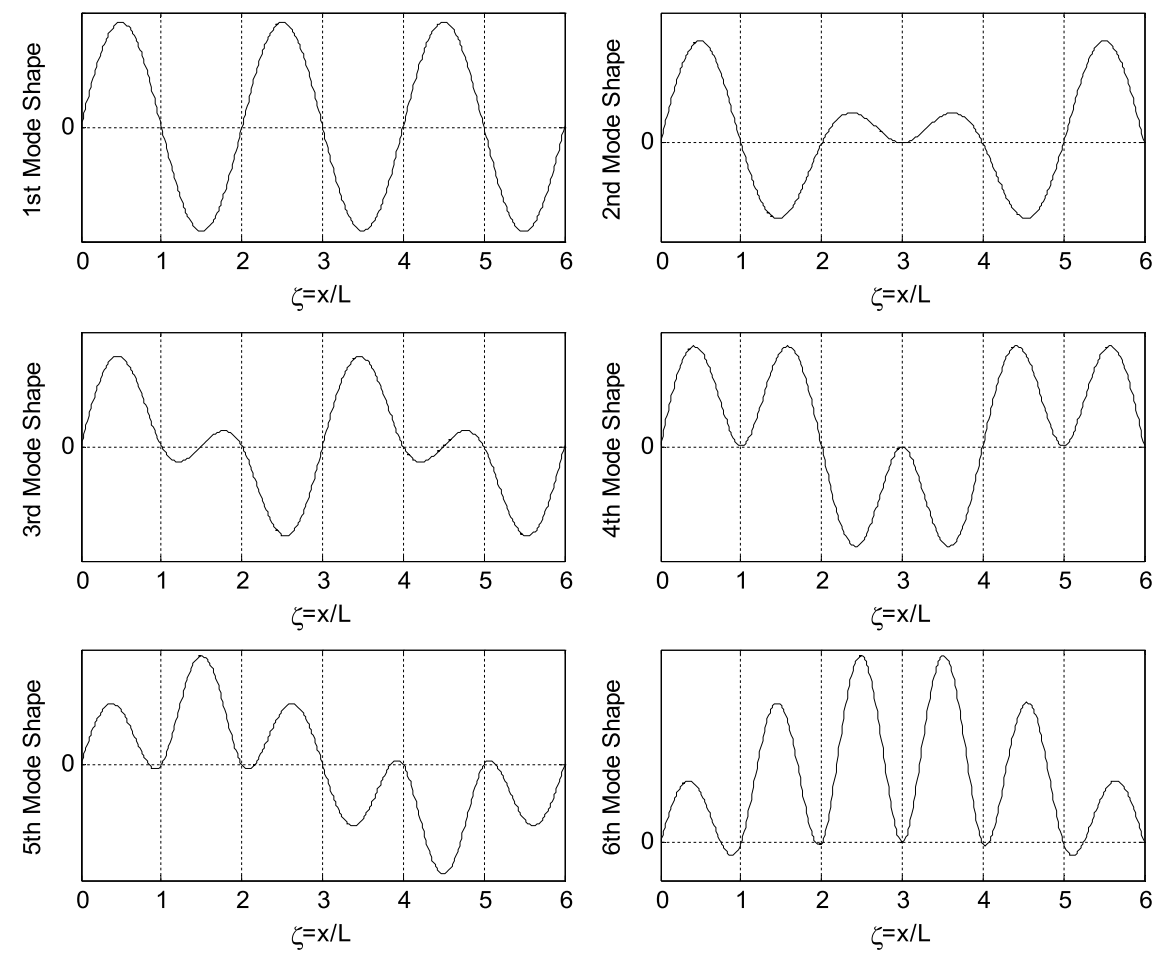

Fig. 4. First cluster of mode shapes of the six-span beam.
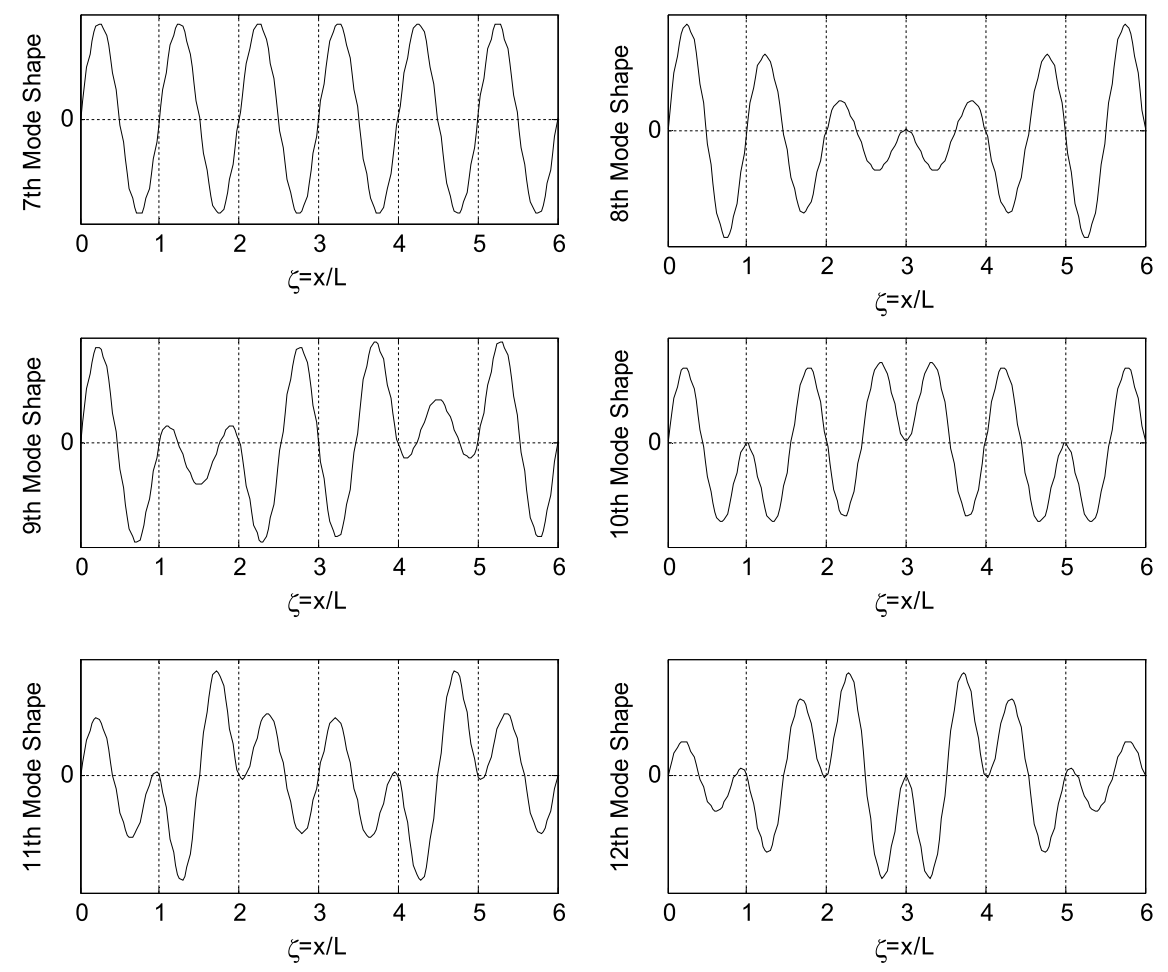

Fig. 5. Second cluster of mode shapes of the six-span beam. 
Table 4

Eigen-vectors for first cluster of six-span beam

\begin{tabular}{|c|c|c|c|c|c|c|c|c|c|}
\hline \multirow[t]{2}{*}{ Mode No. } & \multicolumn{4}{|c|}{ Constants in Eq. (13) } & \multicolumn{5}{|c|}{ Constants in Eq. (15) } \\
\hline & $A_{1}$ & $A_{2}$ & $A_{3}$ & $A_{4}$ & $f_{1}\left(\frac{L}{6}\right)^{3}$ & $f_{2}\left(\frac{L}{6}\right)^{3}$ & $f_{3}\left(\frac{L}{6}\right)^{3}$ & $f_{4}\left(\frac{L}{6}\right)^{3}$ & $f_{5}\left(\frac{L}{6}\right)^{3}$ \\
\hline 1 & 1 & 0 & 0 & 0 & $\frac{1111}{0}$ & 0 & 0 & 0 & 0 \\
\hline 2 & 1 & 0 & 0.009 & 0 & -17.60 & 30.50 & -35.2 & 30.50 & -17.60 \\
\hline 3 & 1 & 0 & 0.0230 & 0 & -74.71 & 74.71 & 0 & -74.71 & 74.71 \\
\hline 4 & 1 & 0 & 0.0279 & 0 & -171.3 & 0 & 171.3 & 0 & -171.3 \\
\hline 5 & 1 & 0 & 0.0246 & 0 & -286.8 & -286.8 & 0 & 286.8 & 286.8 \\
\hline 6 & 1 & 0 & 0.0195 & 0 & -380.7 & -659.5 & -761.6 & -659.6 & -380.8 \\
\hline
\end{tabular}
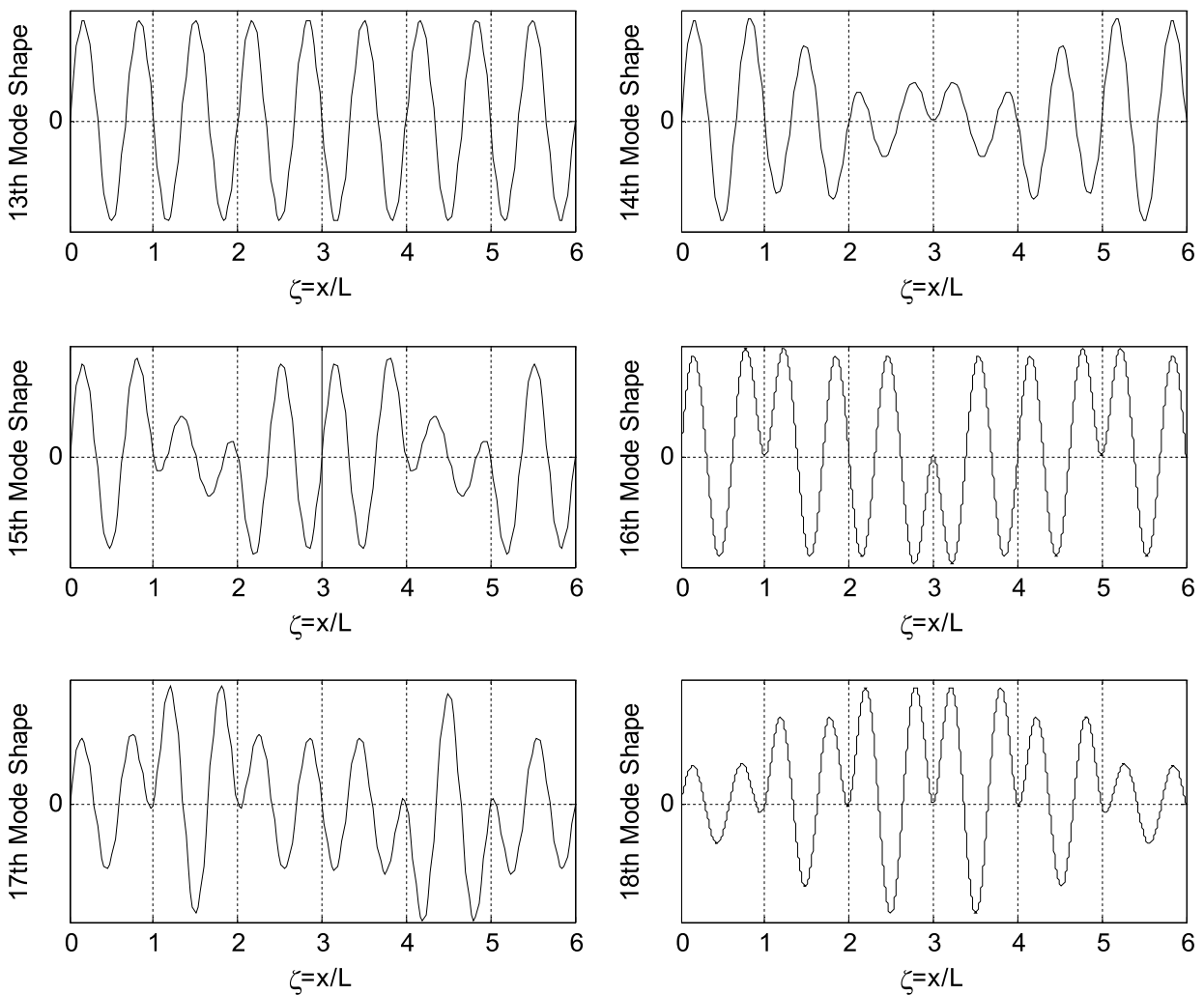

Fig. 6. Third cluster of mode shapes of the six-span beam.

shapes. The bending moment and support force are zero at mid-span of the beam for anti-symmetric modes while the symmetric modes have zero slopes at the mid-span.

In order to study the nature of the homogenous and particular solutions, the eigen-vectors corresponding to the first six natural frequencies are presented in Table 4. As can be seen, the first mode shape is a pure sinusoidal function with no external force at intermediate supports. In other words, in this case the particular solution is zero. Further, the homogenous and particular solutions are plotted in Figs 7 and 8, respectively, for the second mode shape. It can be seen that the hyperbolic functions cause an increase in the magnitude of the homogenous as well as the particular solutions.

The homogenous solution has a variation in the interval $(0,1)$ (see Fig. 7), while the particular solution is zero in the interval $(0,1)$ because of the unit step function (see Fig. 8). Even though the homogenous and particular solutions acquire very large values when $x$ approaches $L$, the sum of the solutions provides finite deflections as can be seen in Fig. 9. 


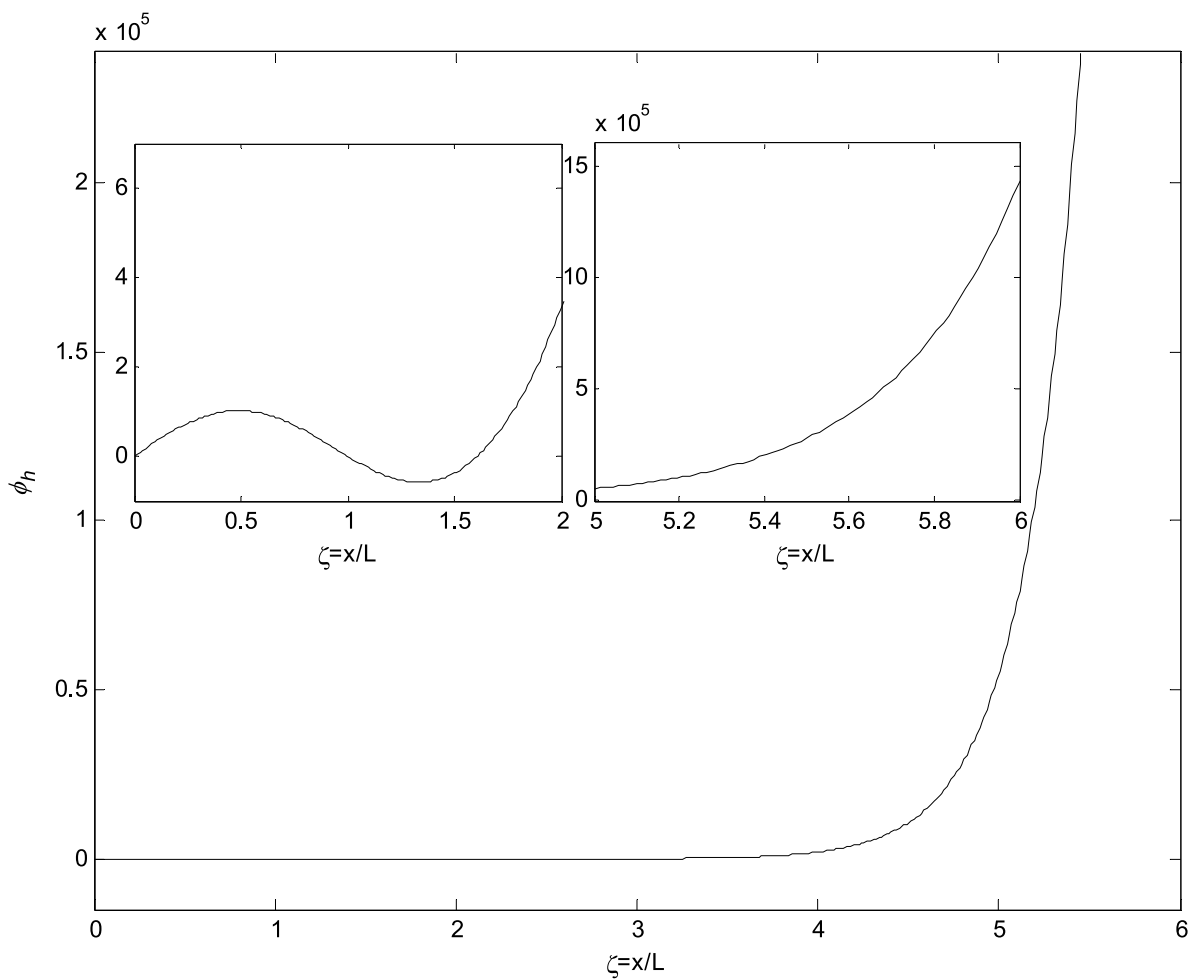

Fig. 7. Homogenous part of the second mode shape for six-span beam.

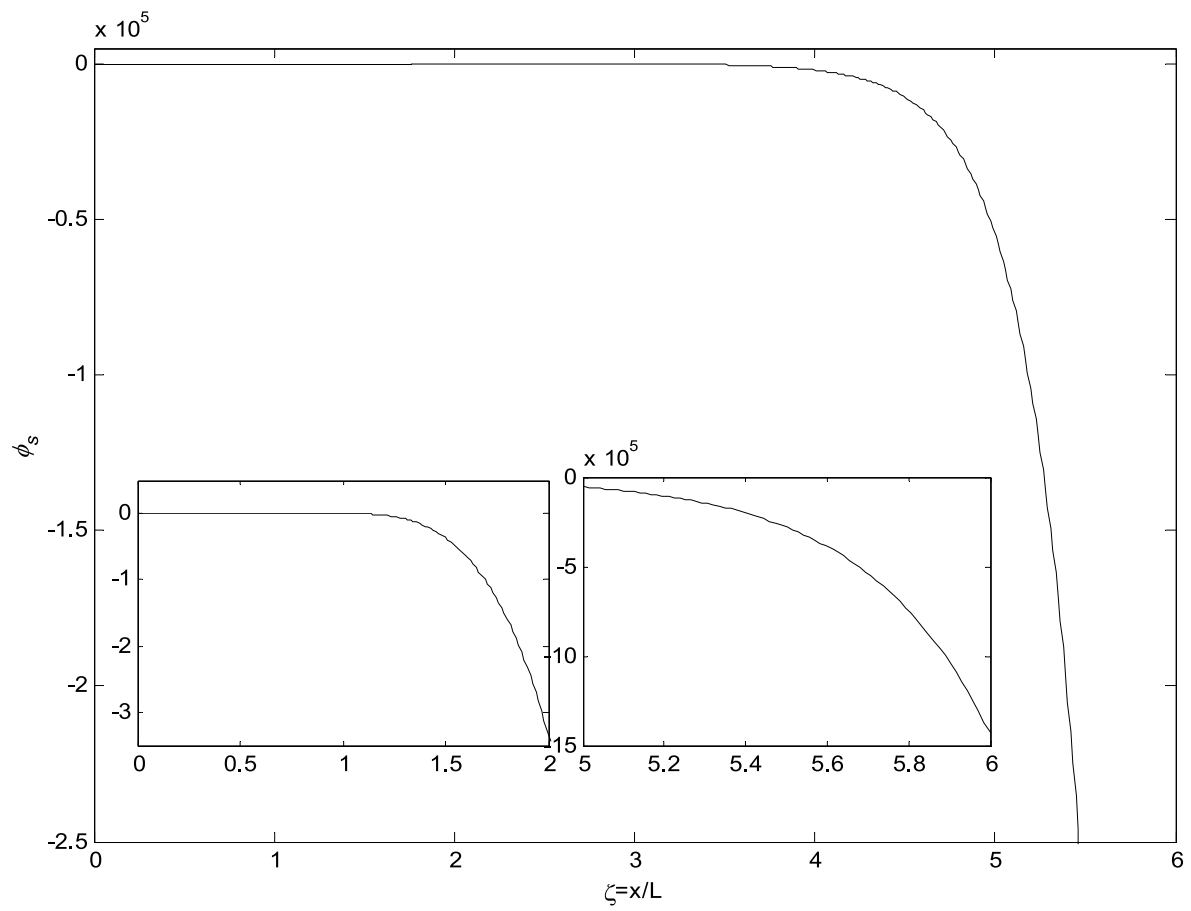

Fig. 8. Particular part of the second mode shape for the six-span beam. 


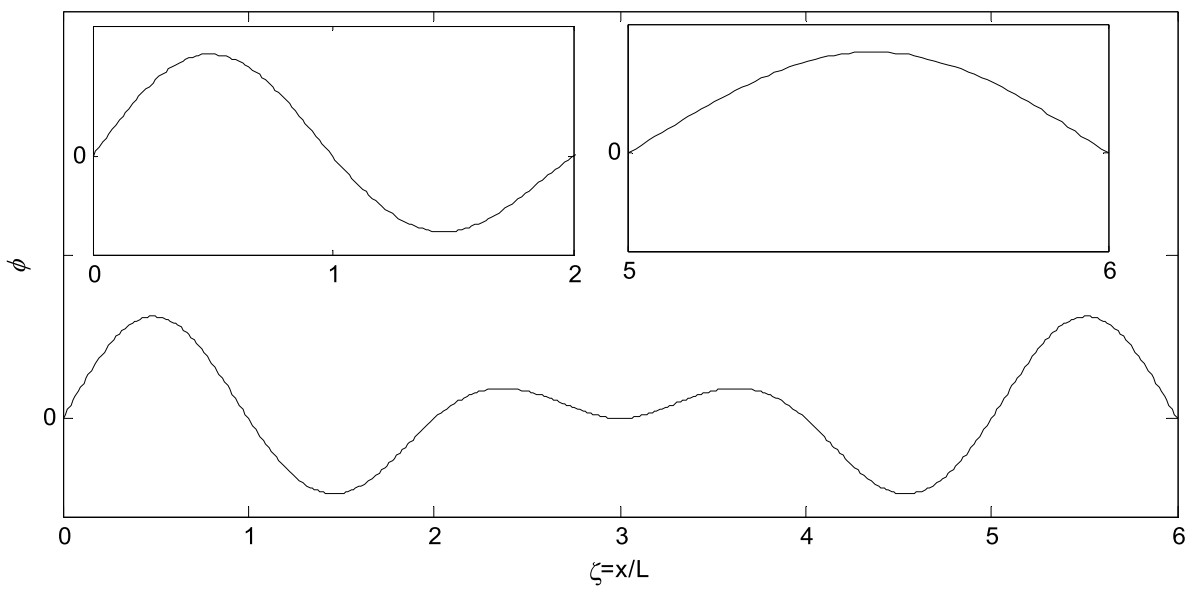

Fig. 9. Second mode shape of the six-span beam.

\subsection{Conclusion}

In this study, a new method is developed to analyse the dynamic behaviour of multi-span beams. The deflection of a multi-span beam is considered as a single function in this method and the constraints except the two ends are replaced by unknown forces. These forces will appear in the eigen-vectors. Hence, the slope conditions and bending moment conditions are being satisfied automatically in the Constrained Characteristic Functions method, and the resulting eigen-value problem is reduced in size. For example, in the third illustrative problem, a $24 \times 24$ matrix eigen-value problem in the conventional method is reduced to a $9 \times 9$ matrix eigen-value problem. Moreover, in Constrained Characteristic Functions, the support reactions of the indeterminate constraints are included in the eigen-problem, and they are obtainable as eigen-vectors, whereas in the conventional method they are obtained by a third spatial differentiation of the mode shapes at the intermediate support positions.

\section{Appendix}

Comparing the conditions of the span-wise characteristic function method and constrained characteristic function method for the six-span beam in case study 3

\begin{tabular}{|c|c|}
\hline Span-wise characteristic function method & Constrained characteristic function method \\
\hline Boundary conditions at two ends: & Boundary conditions at two ends: \\
\hline $\begin{array}{l}\psi_{6}(L)=0 \\
\psi^{I I}(L)=0\end{array}$ & $\begin{array}{ll}\phi(0)=0 & \phi(L)=0 \\
\phi^{I I}(0)=0 & \phi^{I I}(L)=0\end{array}$ \\
\hline Constraints at mid-supports: & Constraints at mid-supports: \\
\hline$\psi_{2}(L / 6)=0$ & $\phi(L / 6)=0 \quad \phi(L / 3)=0 \quad \phi(L / 2)=0$ \\
\hline$\psi_{3}(L / 3)=0$ & $\phi(2 L / 3)=0 \quad \phi(5 L / 6)=0$ \\
\hline$\psi_{4}(L / 2)=0$ & \\
\hline$\psi_{5}(2 L / 3)=0$ & \\
\hline$\psi_{6}(5 L / 6)=0$ & \\
\hline Compatibility condtions at mid-supports: & Compatibility condtions at mid-supports: \\
\hline$\psi_{1}^{I I}(L / 6)=\psi_{2}^{I I}(L / 6)$ & Statisfied, since a single function is considered as the solution \\
\hline$\psi_{2}^{I I}(L / 3)=\psi_{3}^{I I}(L / 3)$ & \\
\hline$\psi_{3}^{I}(L / 2)=\psi_{4}^{I}(L / 2) \quad \psi_{3}^{I I}(L / 2)=\psi_{4}^{I I I}(L / 2)$ & \\
\hline$\psi_{4}^{I}(2 L / 3)=\psi_{5}^{I}(2 L / 3) \quad \psi_{4}^{I I}(2 L / 3)=\psi_{5}^{I I}(2 L / 3)$ & \\
\hline$\psi_{5}^{I}(5 L / 6)=\psi_{6}^{I}(5 L / 6) \quad \psi_{5}^{I I}(5 L / 6)=\psi_{6}^{I I}(5 L / 6)$ & \\
\hline
\end{tabular}




\begin{tabular}{ll}
\hline Span-wise characteristic function method & Constrained characteristic function method \\
\hline Eigen-vector elements: & Eigen-vector elements: \\
$B_{11}, B_{21}, B_{31}, B_{41}$ & $A_{1}, A_{2}, A_{3}, A_{4}$ \\
$B_{12}, B_{22}, B_{32}, B_{42}$ & $f_{1}$ \\
$B_{13}, B_{23}, B_{33}, B_{43}$ & $f_{2}$ \\
$B_{14}, B_{24}, B_{34}, B_{44}$ & $f_{3}$ \\
$B_{15}, B_{25}, B_{35}, B_{45}$ & $f_{4}$ \\
$B_{16}, B_{26}, B_{36}, B_{46}$ & $f_{5}$ \\
\hline
\end{tabular}

\section{References}

[1] Y.K. Lin, Free Vibrations of a Continuous Beam on Elastic Supports, International Journal of Mechanical Sciences 4 (1962), $409-423$.

[2] J.N. Yang and Y.K. Lin, Frequency Response Functions of a Disordered Periodic Beam, Journal of Sound and Vibration 38(3) (1964), 317-340.

[3] Y.K. Lin, I.D. Brown and P.C. Deutschle, Free Vibration of a Finite Row of Continuous Skin-Stringer Panels, Journal of Sound and Vibration 1(1) (1964), 14-27.

[4] D.J. Mead and Y. Yaman, The Harmonic Response of Uniform Beams on Multiple Linear Supports: A Flexural Wave Analysis, Journal of Sound and Vibration 141(3) (1990), 465-484.

[5] T.J. Memory, D.P. Thambiratnam and G.H. Brameld, Free Vibration Analysis of Bridges, Engineering Structures 17(10) (1995), 705-713.

[6] C.H. Loh and B.D. Ku, An Efficient Analysis of Structural Response for Multiple-Support Seismic Excitations, Engineering Structures 17(1) (1995), 15-26.

[7] C.J. Wu and R.G. White, Reduction of Vibrational Power in Periodically Supported Beams by Use of a Neutralizer, Journal of Sound and Vibration 187(2) (1995), 329-338.

[8] M. Ichikawa, Y. Miyakawa and A. Matsuda, Vibration Analysis of the Continuous Beam Subjected to a Moving Mass, Journal of Sound and Vibration 230(3) (2000), 493-506.

[9] X.Q. Zhu and S.S. Law, Orthogonal Function in Moving Loads Identification on a Multi-Span Bridge, Journal of Sound and Vibration 245(2) (2001), 329-345.

[10] H.P. Lin and S.C. Chang, Free Vibration Analysis of Multi-span Beams with Intermediate Flexible Constraints, Journal of Sound and Vibration 281(1-2) (2005), 155-169.

[11] X.Q. Zhu and S.S. Law, Moving Load Identification on Multi-Span Continuous Bridges with Elastic Bearings, Mechanical Systems and Signal Processing 20 (2006), 1759-1782.

[12] S. Naguleswaran, Transverse Vibration of an Euler-Bernoulli Uniform Beam on up to Five Resilient Supports Including Ends, Journal of Sound and Vibration 261(2) (2003), 372-384.

[13] H.Y. Lin and Y.C. Tsai, Free Vibration Analysis of a Uniform Multi-Span Beam Carrying Multiple Spring-Mass Systems, Journal of Sound and Vibration 302(3) (2007), 442-456.

[14] Y. Yesilce and O. Demirdag, Effect of Axial Force on Free Vibration of Timoshenko Multi-Span Beam Carrying Multiple Spring-Mass Systems, International Journal of Mechanical Sciences 50(6) (2008), 995-1003.

[15] Y. Mikata, Orthogonality Condition for a Multi-Span Beam, and its Application to Transient Vibration of a Two-Span Beam, Journal of Sound and Vibration 314(3-5) (2008), 851-866. 

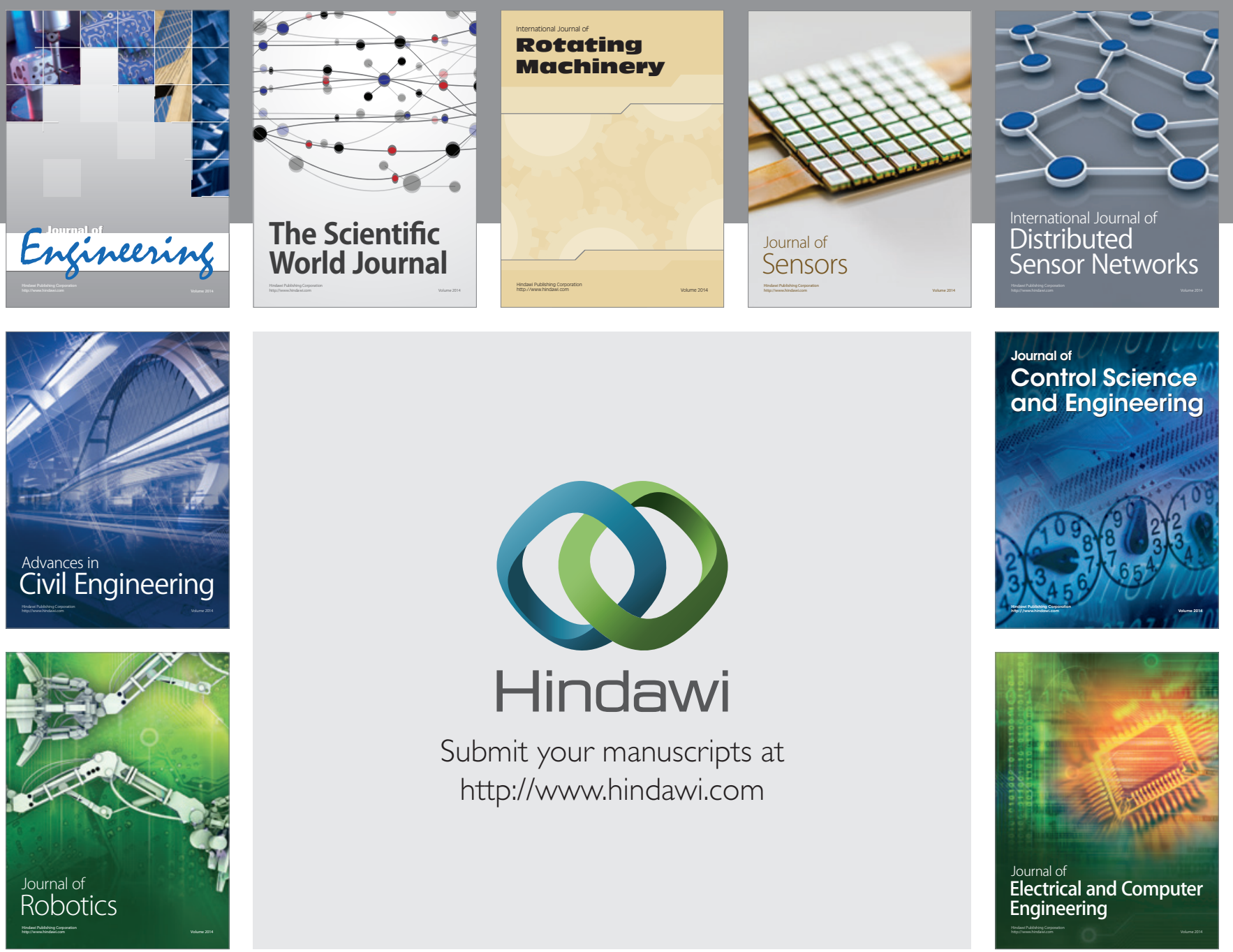

Submit your manuscripts at

http://www.hindawi.com
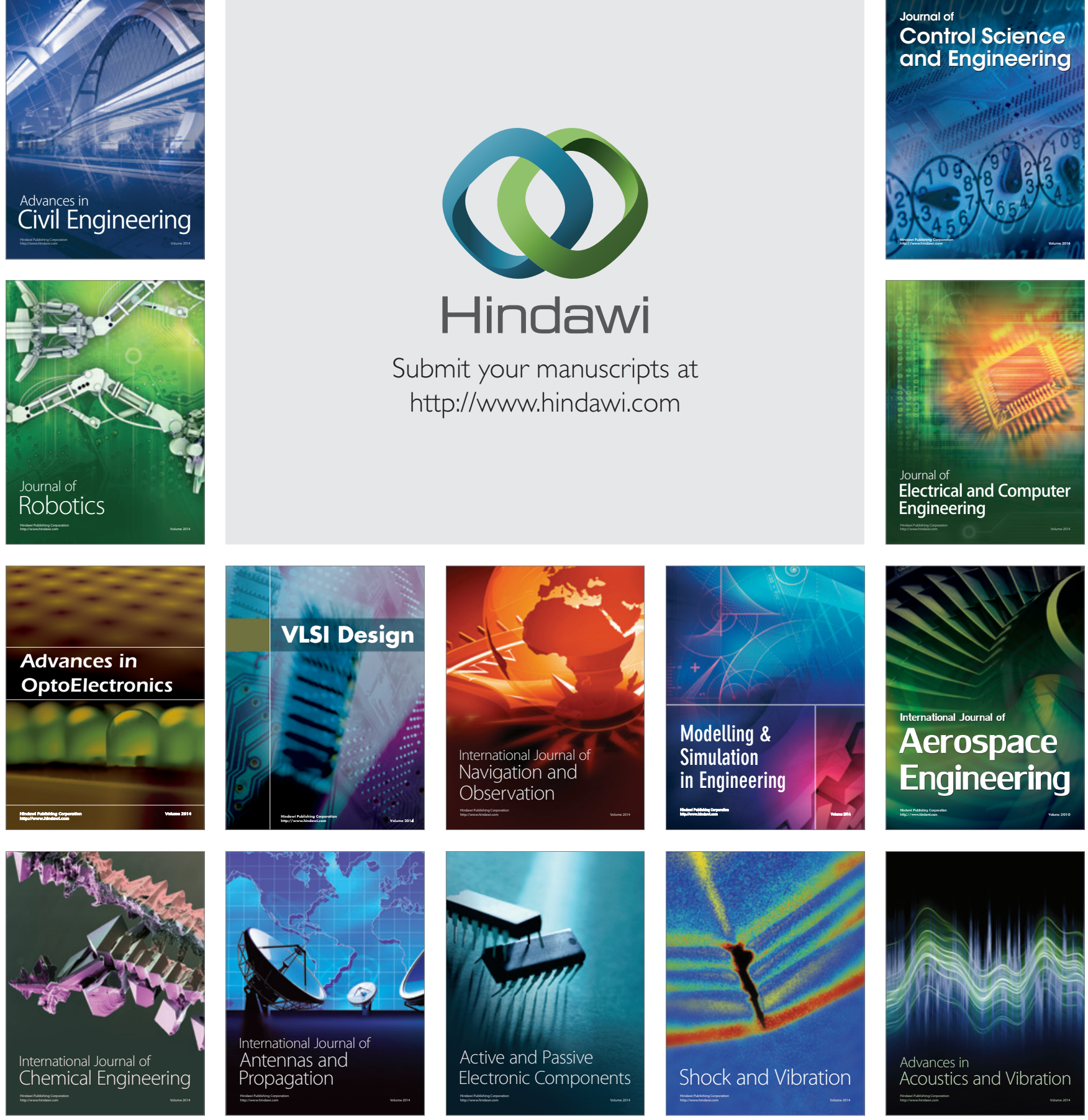